\title{
EVOLUTION OF PILED-UP COMPRESSIONS IN MODELED CORONAL MASS EJECTION SHEATHS AND THE RESULTING SHEATH STRUCTURES
}

\author{
Indrajit Das ${ }^{1}$, Merav Opher ${ }^{1,2}$, Rebekah Evans $^{1}$, Cristiane Loesch $^{3}$, and Tamas I. Gombosi $^{4}$ \\ ${ }^{1}$ George Mason University, 4400 University Drive, Fairfax, VA 22030, USA; idas@masonlive.gmu.edu, revansa@gmu.edu \\ ${ }^{2}$ Astronomy Department, Boston University, 725 Commonwealth Avenue, Boston, MA 02215, USA; mopher@bu.edu \\ ${ }^{3}$ Laboratorio Associado de Plasma, Instituto Nacional de Pesquisas Espaciais (INPE), Avenida dos Astronautas 1758, Jardim da Granja, Sao Jose dos Campos, \\ SP 12227-010, Brazil; crisloesch@gmail.com \\ ${ }^{4}$ University of Michigan, 1517 Space Research Building Ann Arbor, MI 48109, USA; tamas@umich.edu \\ Received 2010 August 18; accepted 2010 December 2; published 2011 February 16
}

\begin{abstract}
We study coronal mass ejection (CME)-driven shocks and the resulting post-shock structures in the lower corona $\left(2-7 R_{\odot}\right)$. Two CMEs are erupted by modified Titov-Démoulin (TD) and Gibson-Low (GL) type flux ropes (FRs) with the Space Weather Modeling Framework. We observe a substantial pile-up of density compression and a narrow region of plasma depletion layer (PDL) in the simulations. As the CME/FR moves and expands in the solar wind medium, it pushes the magnetized material lying ahead of it. Hence, the magnetic field lines draping around the CME front are compressed in the sheath just ahead of the CME. These compressed field lines squeeze out the plasma sideways, forming PDL in the region. Solar plasma being pushed and displaced from behind forms a strong piled-up compression (PUC) of density downstream of the PDL. Both CMEs have comparable propagation speeds, while GL has larger expansion speed than TD due to its higher initial magnetic pressure. We argue that high CME expansion speed along with high solar wind density in the region is responsible for the large PUC found in the lower corona. In case of GL, the PUC is much wider, although the density compression ratio for both the cases is comparable. Although these simulations artificially initiate out-of-equilibrium CMEs and drive them in an artificial solar wind solution, we predict that PUCs, in general, will be large in the lower corona. This should affect the ion profiles of the accelerated solar energetic particles.
\end{abstract}

Key words: magnetohydrodynamics (MHD) - Sun: corona - Sun: coronal mass ejections (CMEs)

Online-only material: color figures

\section{INTRODUCTION}

Coronal mass ejections (CMEs) are one of the most violent and energetic phenomena in the solar system. On an average, $10^{15}-10^{16} \mathrm{~g}$ of plasma are ejected from the solar corona. CMEs propagate with speeds ranging from 50 to $2500 \mathrm{~km} \mathrm{~s}^{-1}$ while they can also expand. Here we explore the effects of CME expansion inside its sheath structure. Particularly, we explore the conditions for the formation of pile-up of density and plasma depletion layer (PDL) in two different simulations of out-ofequilibrium CMEs. The expansion of a CME could occur for several reasons (Gosling et al. 1998): first, at the time of its ejection, the CME's leading edge could move faster than its trailing edge; second, its initial internal pressure could exceed the ambient solar wind pressure resulting in CME expansion. The higher internal pressure can be a result of a higher thermal or magnetic pressure. We expect that the expansion rates of the CMEs will decrease with the increasing heliocentric distance, while the CME continues to interact with the surrounding solar wind. Besides the initial internal pressure, the external pressure gradient can also be important. Démoulin \& Dasso (2009) have shown that the main driver of a magnetic cloud's (MC) expansion is the rapid decrease of the total solar wind pressure with distances from the Sun. Gosling et al. (1998) reported that simulated CMEs can be found to expand until about $6 \mathrm{AU}$.

Dasso et al. (2007) reported that MCs have significantly higher velocity in their front than in their back which clearly shows that they expand. Dal Lago et al. (2003) reported that CME expansion speed can be more than $1800 \mathrm{~km} \mathrm{~s}^{-1}$. Interestingly, Gulisano et al. (2010) reported a broad range of MC expansion velocities of $\sim 80-500 \mathrm{~km} \mathrm{~s}^{-1}$ between 0.3 and $1.0 \mathrm{AU}$ in which the expansion velocity decreases only weakly. Dal Lago et al. (2003) proposed an empirical relation of $V_{R}=0.88 \times V_{\mathrm{EX}}$ using 57 limb CMEs observed by LASCO and EIT from 1997 January to 2001 April, where $V_{\mathrm{EX}}$ is the CME expansion speed and $V_{R}$ its radial propagation speed.

Siscoe \& Odstrcil (2008) have discussed the implications of the propagation and expansion of CMEs/ICMEs. They found that, if the deflection of the solar wind near the leading edge of an ICME is not large enough to let it flow around the body of the ICME, the solar wind piles up around its face. As the CME/ICME expands and propagates faster than the laterally deflected flow, the solar wind is expected to pile up in front of the nose of the object. In our simulation, we observe a strong pile-up compression or piled-up compression (PUC) of density in the lower corona. We show that this happens primarily due to the strong CME expansion in case of GL and propagation in the case of TD.

There have been observations of mass pile-up in front of a CME/ICME at 1 AU. Dasso et al. (2007) reported an ICME event of 2004 November 9-11 observed by the Wind spacecraft in which they report, "The ICME is preceded by typical pile-up of solar wind material. This corresponds to plasma and magnetic field pushed from behind the ICME, forming the turbulent preICME sheath...." Mostl et al. (2009) looked at the internal structures of a CME at $1 \mathrm{AU}$ through white light images in a case study (2008, June 6-7) and found two density peaks on either side of a plasma void or dark cavity region. The plasma void or dark cavity region is where the magnetic flux rope (MFR) lies. They speculated that these double density peaks bracketing 
the MFR rise from the material originating from the corona and the solar wind being swept up by the CME in the sheath. We can also find similar plasma piled-up structures around the MCs in Rouillard et al. (2010) and Lynch et al. (2010). Farrugia et al. (2008) identified a higher plasma pressure region in front of a MC at $1 \mathrm{AU}$ with a two-dimensioanl magnetohydrodynamic (MHD) code. They argued that this increase in pressure is due to the plasma pile-up ahead of the MC which occurs because of the expansion of the ejecta.

In constructing the theoretical model of the PDL, Zwan \& Wolf (1976) explained the plasma squeezing out process along the interplanetary magnetic field lines in case of magnetosphere bow shock. Erkaev et al. (1995) developed an MHD theory to analyze the "magnetic barrier" or the "depletion layer" in case of interplanetary shocks driven by MCs for both steady and nonsteady situations. Farrugia et al. (1997) observed a PDL ahead of an interplanetary magnetic cloud and explained it with the help of the aforementioned Erkaev et al. (1995) model. Liu et al. (2006) also observed that PDLs can be found in the sheath region ahead of a fast moving and expanding $\mathrm{MC}$ at the location where magnetic field lines drape around it. As the draped magnetic field lines get compressed by the CME motion inside the sheath, the plasma in the region squeezed along the field lines creating a PDL in the region. Kaymaz \& Siscoe (2006) talked about the draping pattern of magnetic field lines in ten different ICME sheaths produced by fast ICMEs at different longitudes.

The organization of the paper is as follows. In the next section, we describe the details of the CME simulations. Then we present the results for PUCs and PDLs and, lastly, discuss conditions for their formations.

\section{METHODOLOGY FOR CME SIMULATIONS}

\subsection{Background Solar Wind}

We used the Space Weather Modeling Framework (Toth et al. 2005 ) code to produce the background solar wind. It is a threedimensional MHD code with the Block Adaptive Tree Solar Wind Roe Upwind Scheme (BATS-R-US) serving as its core. Different components of the heliosphere with different physics models have been included in this code in a modular fashion and they are coupled with each other. It is highly parallelized with adaptive mesh refinement capability. The initial solar wind solution is generated with the Wang-Sheeley-Arge model with a spatially variable polytropic index $(\gamma)$ mimicking the heating mechanism in the Sun. The effective $\gamma$ is close to 1.0 at the lower boundary and equal to 1.5 above at $12 R_{\odot}$. The gamma profile has been fixed to limit the exchange of heat between the CME and the background solar wind after the FR was inserted into the background. Evans et al. (2009) calculated the amount of background heating due to the variable $\gamma$. We used the same background solar wind as used by Liu et al. (2008) and Evans et al. (2011). The code creates the initial solar magnetic field using the Potential Field Source Surface Model and we used the magnetogram for CR1922 (Cohen et al. 2007) for this simulation. This particular rotation corresponds to 1997 April-May (solar minimum). A steady state solution has been reached after 15,000 time steps, generating fast solar wind at high latitudes and slow wind at the low latitudes.

\subsection{CME Initiation}

A high-resolution $\left(3 / 256 R_{\odot}\right)$ box is placed in the path of the $\mathrm{CME}$ after the steady state solution is reached. The rectangular box stretches $1 R_{\odot}$ in longitude, $1.8 R_{\odot}$ in latitude, and $7 R_{\odot}$ in the radial direction. We refine the location along the current sheet with $3 / 32 R_{\odot}$ sized cells and there are $12.4 \times 10^{6}$ number of cells in the $24 \times 24 \times 24 R_{\odot}$ simulation box.

On top of this steady state solar wind solution, in an active region near the equator (NOAA AR8038), a modified TD FR (Titov \& Démoulin 1999; Roussev et al. 2004) has been inserted out-of-equilibrium. This active region was chosen to be the same as the source region for the 1997 May $12 \mathrm{CME}$, which was directed toward the Earth (Thompson et al. 1998). This modified TD FR contains only poloidal magnetic field. The parameters for the modified TD FR are chosen to be: torus radius $95 \mathrm{Mm}$, cross section radius $18 \mathrm{Mm}$, mass $4.5 \times 10^{12} \mathrm{~g}$, and torus current $5 \times 10^{11} \mathrm{~A}$. Initial free energy and initial density for TD FR have been estimated to be $\sim 2 \times 10^{32}$ erg and $\sim 1.5 \times 10^{-17} \mathrm{~g} \mathrm{cc}^{-1}$. For GL (for details, see Gibson \& Low 1998 and Manchester et al. 2004, 2005), the initial FR was specified by the nondimensional parameters: $a=0.7$, stretching the length of the FR into a teardrop shape in the radial direction; $r_{0}=0.75$, the FR radius; $r_{1}=1.8$, the distance from the solar center where the FR is placed (prior to the radial contraction); $a_{1}=0.93$, the free parameter that determines the magnetic field strength and plasma pressure in the FR (Loesch et al. 2011). This choice gives the GL FR a toroidal magnetic component with an initial magnetic energy of $9.0 \times 10^{32}$ erg estimated by subtracting the steady state magnetic energy from the total magnetic energy of the $\mathrm{CME}$ at its beginning. For both the initiation mechanisms, the initial magnetic energies drive the CMEs. Both the FRs have the same footpoint separation and are centered at the same position $\left(x=1.08 R_{\odot}, y=0.27 R_{\odot}, z=0.11 R_{\odot}\right)$.

\section{RESULTS}

\subsection{Piled-up Density Compression in the Sheath}

In Figure 1, we present structures associated with $\mathrm{CME}$ propagations: the FR, shock surface, sheath, and PUC on the $Y=0.4 R_{\odot}$ plane for TD at 48 minutes and GL for 30 minutes. The shocks for both the cases are at a distance of $4.6 R_{\odot}$ from the Sun along the white straight line shown in Figure 1. This line passes close to the nose of the CME shocks and also follows the region of highest numerical resolution of $3 / 256 R_{\odot}$. Below, we analyze the data collected along this line. We use contours of $\theta_{B}$ to locate the FR (Evans et al. 2011), where, $\theta_{B}=\sin ^{-1}\left(\frac{B_{N}}{B}\right)$, $B$ being total magnetic field and $B_{N}$ being magnetic field along $\vec{N} . \vec{R}$ gives the radial direction away from the Sun, $\vec{Z}$ points toward solar rotation axis, $\vec{T}=\vec{Z} \times \vec{R}$ and $\vec{N}=\vec{R} \times \vec{T} . \theta_{B}$, therefore, represents the orientation of the magnetic field. Flux ropes having their own magnetic fields should have different field orientations than the solar wind. Hence, following the changes in $\theta_{B}$, we can detect the changes in magnetic field orientations and locate the flux rope. This definition has been similarly used in Burlaga (1988). In Figures 1(a) and (c), we can observe the contours of $\theta_{B}$ for both GL and TD, respectively, to locate the flux rope following their sudden changes.

The flux rope while moving in the solar wind medium pushes and displaces the solar wind plasma, which piles up. This PUC, or what we called the PUC, can be seen in Figures 1(b) and (d) for both GL and TD, respectively. In Figures 1(b) and (d), we plotted $r_{\text {diff }}$, where $r_{\text {diff }}=$ (solar wind density at any instant of CME propagation) - (background solar wind density) for the corresponding CMEs. We can see that in front of the flux rope there is a region of enhanced density.

Figure 2 shows the detailed features of CME and its sheath structures for both TD and GL simulations along the white 

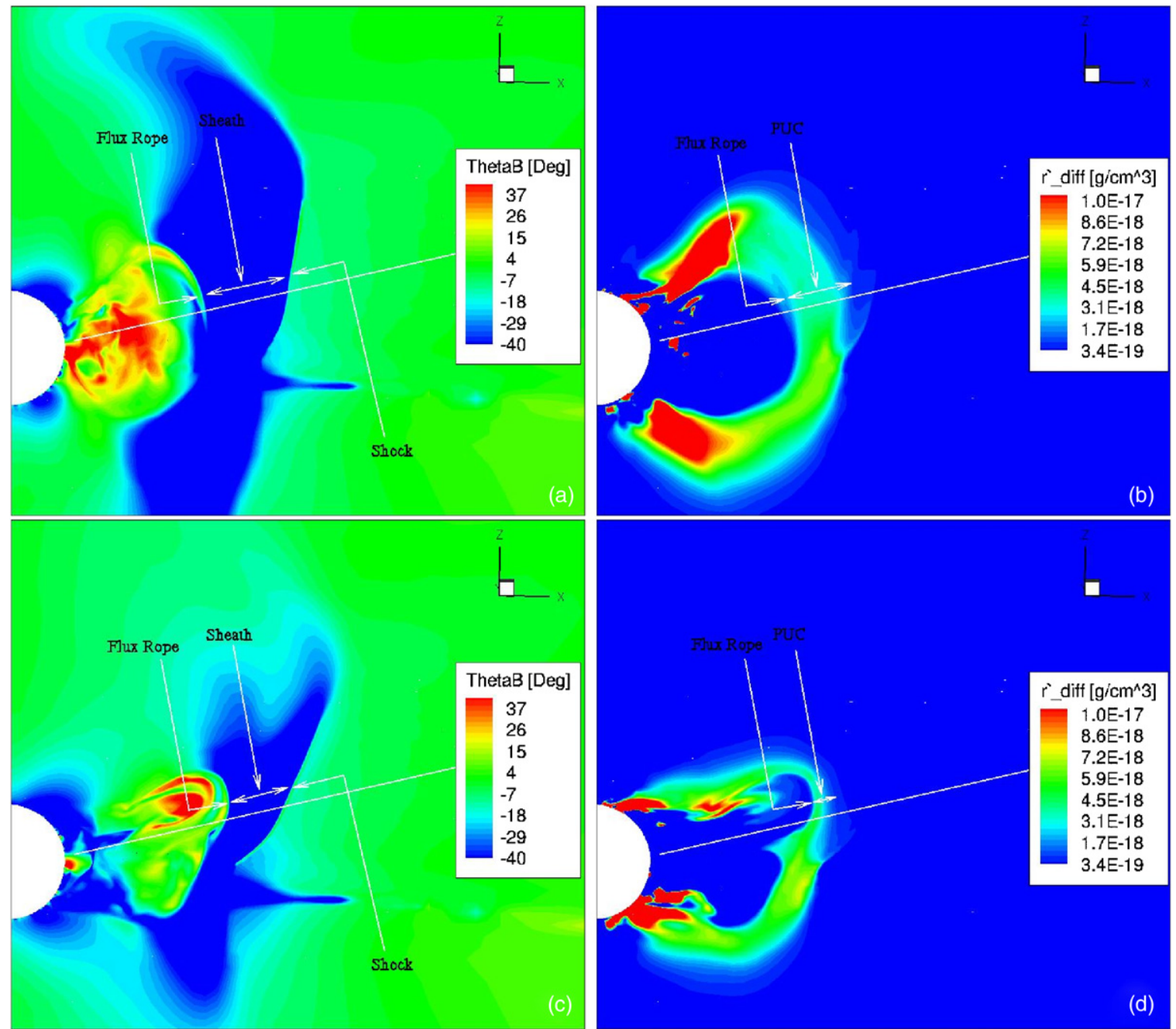

Figure 1. Two-dimensional contour plots at $Y=0.4 R_{\odot}$ plane showing flux rope, shock, and sheath. Top row from left: (a) contours with $\theta_{B}$ and (b) with $r_{\text {diff }}$ for GL at 30 minutes where, $\theta_{B}=\sin ^{-1}\left(\frac{B_{N}}{B}\right), B=$ total magnetic field, $B_{N}=$ magnetic field along $\vec{N}$. $\vec{R}$ gives the radial direction away from the Sun, $\vec{Z}$ points toward solar rotation axis, $\vec{T}=\vec{Z} \times \vec{R}$ and $\vec{N}=\vec{R} \times \vec{T}$. We can see that $\theta_{B}$ is a very useful tool to locate the flux rope (Evans et al. 2011); $r_{\text {diff }}=$ (solar wind density at 30 minutes) - (background solar wind density), showing only the "positive" part of the contour. Background solar wind density is the density of the solar wind before the CME was inserted. Bottom row from left: (c) contour with $\theta_{B}$ and (d) with $r_{\text {diff }}$ for TD at 48 minutes. Following the white line shown in the plots, we collect and analyze the data in the plots to be followed.

(A color version of this figure is available in the online journal.)

line shown in Figure 1. We can see the flux rope is followed by a PDL and then the PUC outlined in light yellow shaded region. The formation of the PDL will be explained in the next section. Magnetic pressure, $p B_{\mathrm{cme}}$, has been calculated by subtracting the steady state magnetic pressure from the total magnetic pressure at any particular instant of time. This subtraction has been done at $Y=0.4 R_{\odot}$ plane at any given time. The compression ratio for PUC along this line, $\rho_{\text {PUC }} / \rho_{b}$ is $\sim 6-10$ times than that for the compression ratio for forward shock (note that the compression ratio is larger than 4 because the polytropic index $\gamma<5 / 3) \rho_{\text {shock }} / \rho_{b}$, where $\rho_{\text {PUC }}$ is the peak density in PUC, $\rho_{\text {shock }}$ is the jump in density in the shock, and $\rho_{b}$ is the background solar wind density at the corresponding locations for TD and GL cases.

The pile-up should occur if $V_{\mathrm{EX}}+V_{P}=V_{\mathrm{LE}}>V_{D}$ (Siscoe \& Odstrcil, 2008) criteria is satisfied, where, $V_{P}$ and $V_{\mathrm{LE}}$ are, respectively, the propagation and leading edge speeds of the CME and $V_{D}$ is the deflection speed of the solar wind in the CME sheath. We estimate $V_{P}$ and $V_{\mathrm{EX}}$ for the flux rope along the white lines shown in Figure 1 for both TD and GL cases, while the deflection speed is taken as the solar wind speed in the sheath along the same line. $V_{\mathrm{EX}}$ has been estimated by tracking the increase of the flux rope radii at different times and $V_{P}$ by looking at the speeds at the tip of the flux rope along the white line shown in Figure 1. All speeds are in the solar wind frame measured on the $Y=0.4 R_{\odot}$ plane. Figures 3(a) and (b) present how $V_{\mathrm{EX}}, V_{P}$, and $V_{D}$ vary with shock distance from the Sun for both TD and GL. We find both TD and GL to have comparable propagation speeds, while $V_{\mathrm{EX}}$ is a factor of 2-5 larger for GL than that in TD. This is probably due to the higher initial magnetic energy of GL than TD (Loesch et al. 2011). As $V_{P}$ and $V_{D}$ are comparable for both GL and TD cases, $V_{\mathrm{EX}}$ becomes the main driver of the PUC. As $V_{\mathrm{EX}}$ is much larger for GL than in TD, we expect GL to have a larger PUC. In Figure 4(a), we plot $V_{\mathrm{NET}}=V_{\mathrm{LE}}-V_{D}$ with distance from the Sun. $V_{\mathrm{NET}}$, and hence the PUC, is being controlled by $V_{\mathrm{LE}}$. Figure $4(\mathrm{~b})$ shows the evolution of PUC calculated on the $Y=0.4 R_{\odot}$ plane. Figure 4 (c) presents the compression ratio in PUC, $\rho_{\text {PUC }} / \rho_{b}$, and forward shock, $\rho_{\text {shock }} / \rho_{b}$, as a function of shock distance. We can see that as $V_{\mathrm{NET}}$ increases, $\rho_{\mathrm{PUC}} / \rho_{b}$ 

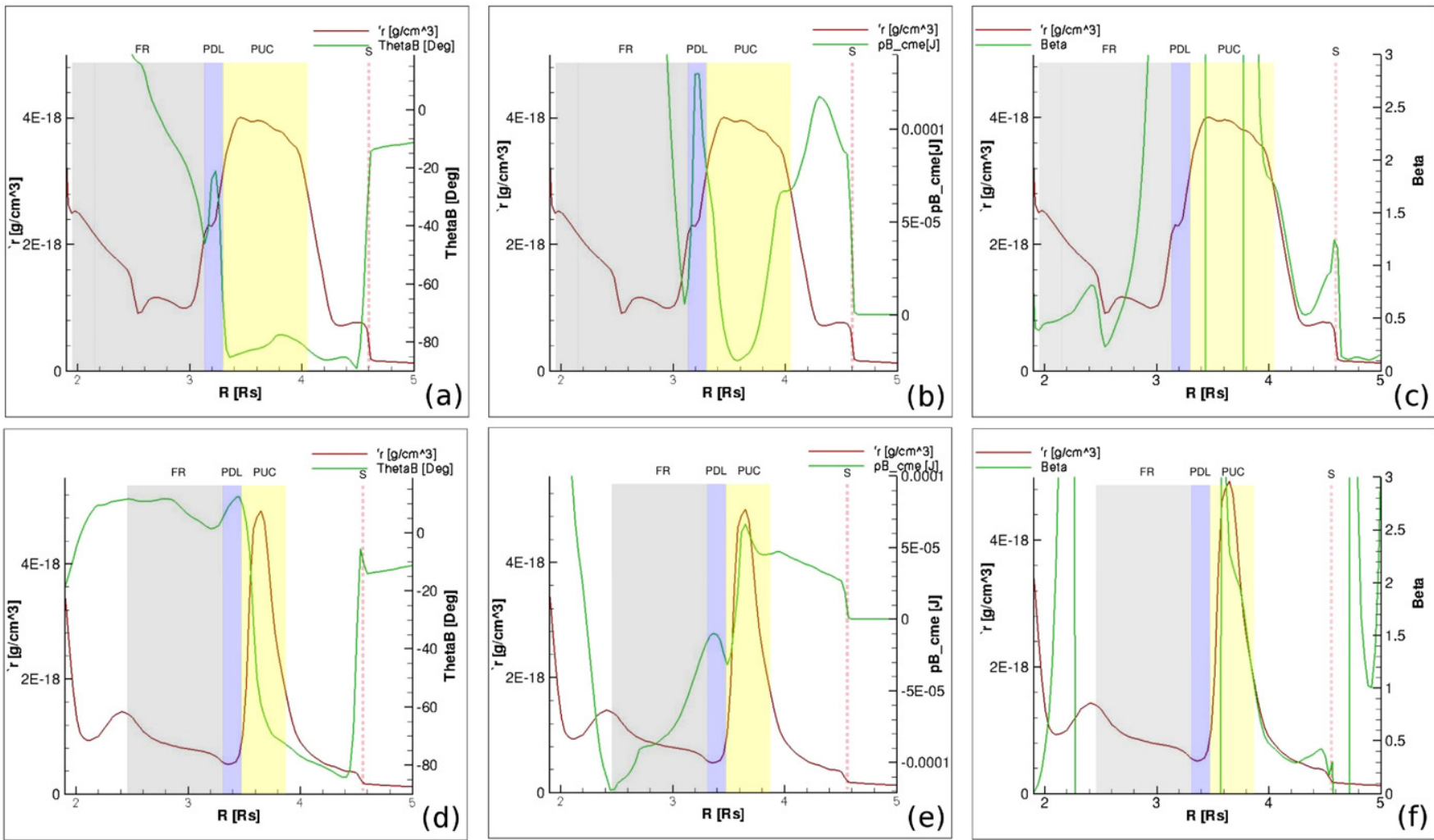

Figure 2. One-dimensional plots showing locations of the flux rope (FR) with light gray, PDL with light blue, PUC with light yellow shaded regions, respectively, while shock (S) is shown with light red dash-shaded regions when shock is at $\sim 4.6 R_{\odot}$ along the white line shown in Figure 1 for GL at 30 minutes and TD at 48 minutes. Top row from left: plots for (a) density and $\theta_{B}$, (b) density and magnetic pressure ( $\left.p B_{\text {cme }}\right)$, and (c) density and plasma beta $(\beta)$ for GL. Bottom row from left: plots for (d) density and $\theta_{B}$, (e) density and $p B_{\mathrm{cme}}$, and (f) density and $\beta$ for TD. The shocks are at about the same distances from the Sun along the line shown in Figure 1.

(A color version of this figure is available in the online journal.)
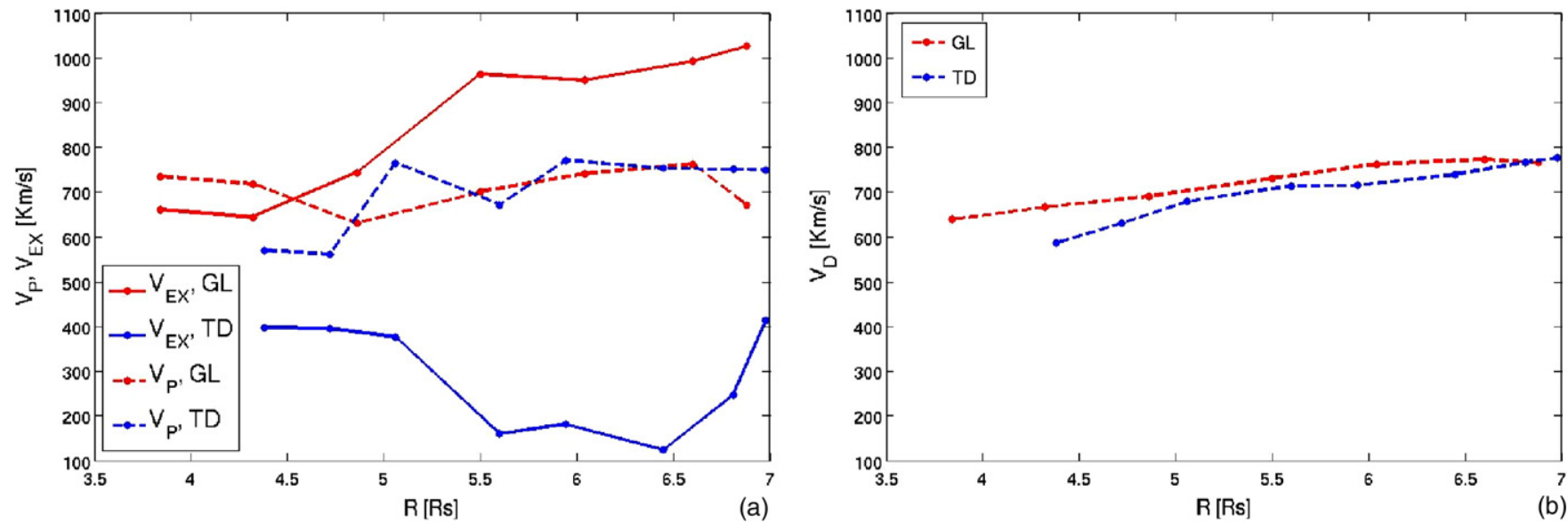

Figure 3. Variations of (a) $V_{\mathrm{EX}}$, expansion speed and $V_{P}$, propagation speed of CME, and (b) $V_{D}$, the deflection speed of solar wind inside the sheath, with shock distance from the Sun for both TD and GL initiation mechanisms estimated along the white line shown in Figure 1.

(A color version of this figure is available in the online journal.)

also goes up. Figure 4(d) presents the variation of sheath width and the PUC width with shock distance from the Sun. We can observe a general increase in both sheath and PUC widths for both the simulations even though GL has a higher rate of increase than TD. Also, the sheath and PUC widths are larger for GL than in TD, and PUC occupies almost all the width of the sheath for GL. As we already mentioned above, GL is a larger CME with higher initial magnetic energy (Loesch et al. 2011) and expansion speeds. Therefore, it has wider sheath and PUC structures. Figure 5 shows a synthetic line-of-sight coronagraph plot for TD at 42 minutes. We have integrated the density values along the line of sight from the $X-Z$ plane. The contour values have been calculated as the ratio of the total integrated density, along that line of sight, toward that location on the plane, to the background density at the corresponding location when no CME was present. Therefore, this ratio, called the WL Ratio, gives us an estimate of the enhancement of density along the line of sight because of the PUC. The black disk mimics the occulting disk 

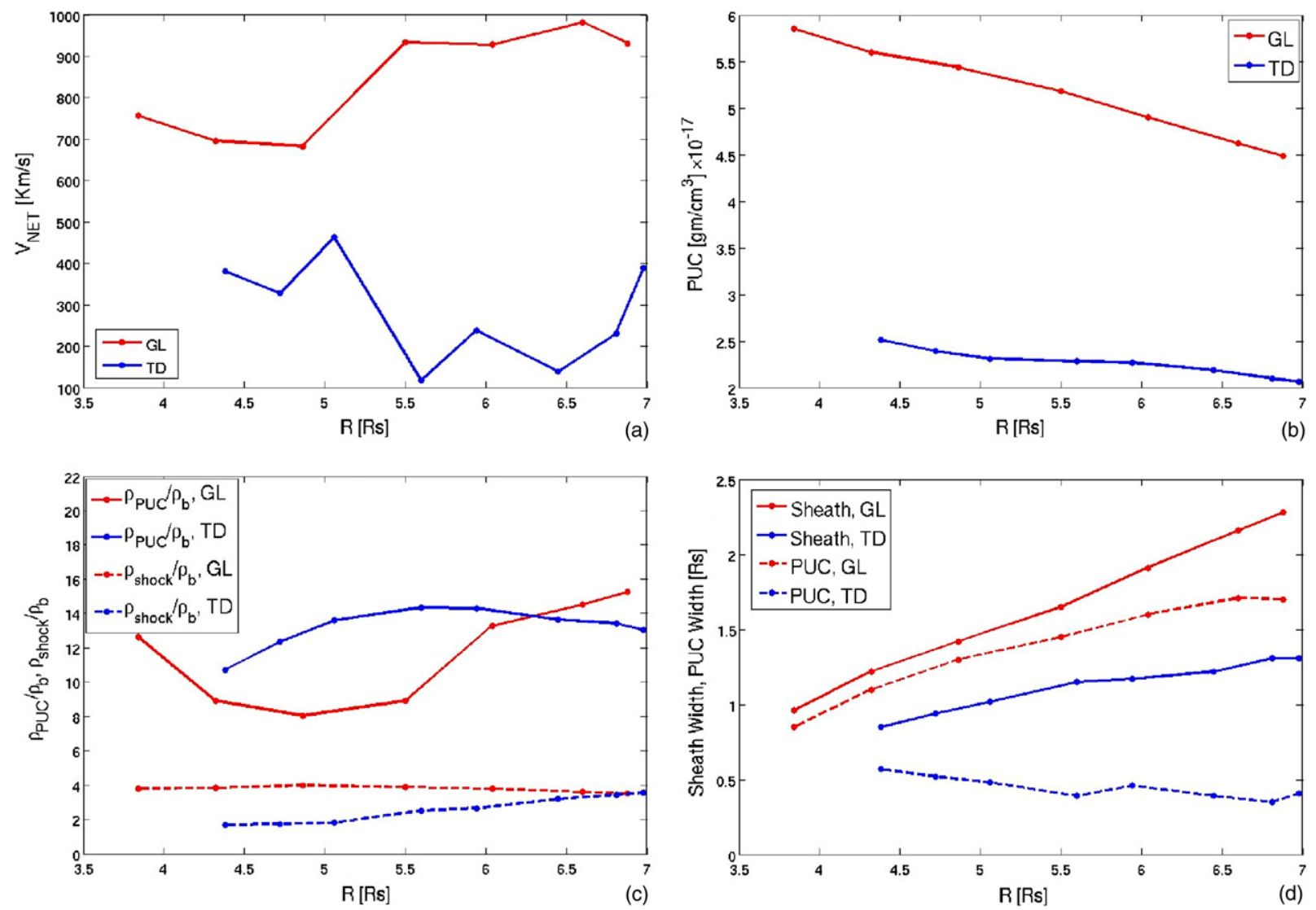

Figure 4. Variations of (a) $V_{\mathrm{NET}}=V_{\mathrm{EX}}+V_{P}-V_{D}$ and (b) PUC (top row from left), and (c) PUC and shock density compression ratio and (d) sheath and PUC widths with shock distance from the Sun following the white line shown in Figure 1 for GL and TD cases (bottom row from left).

(A color version of this figure is available in the online journal.)

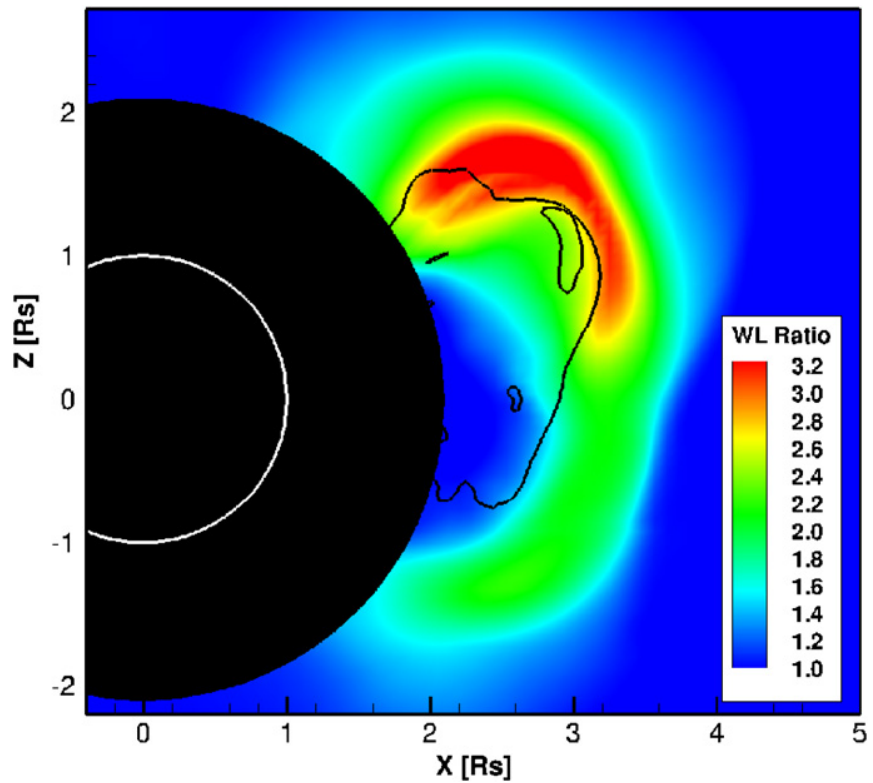

Figure 5. Synthetic white light coronagraphic image at 42 minutes for the TD simulation. The black line locates the CME flux rope on the $X-Z$ plane. The black disk shows the blocked portion of the Sun. The WL Ratio at any location is defined as the ratio between its density integrated along the line of sight at that time and background density at the corresponding location when no CME was present.

(A color version of this figure is available in the online journal.) present in coronagraphic images. The black line shows where the location of the flux rope is in the $X-Z$ plane. The density enhancements ahead of the flux rope (although integrated not just in the $X-Z$ plane) clearly shows as the PUC.

\subsection{Formation of a Plasma Depletion Layer (PDL) in the Sheath}

In Figure 6, adopted from Zwan \& Wolf (1976), we show how the solar wind magnetic flux tubes get compressed by the magnetosphere bow shock. In the figure, solar wind magnetic flux tubes arrive from the left and slam onto the bow shock. Thus, the flux tubes themselves get compressed and hence the plasma inside the flux tubes and outside of it get squeezed out and move along the field lines. Therefore, we get a layer with low density of plasma in and around the compressed field lines. In our simulations, PDLs can be observed in Figure 2 in the light blue shaded regions for GL at 30 minutes and TD at 48 minutes. We show in one-dimensional plots for both the simulations that PDLs have high magnetic pressures as the field lines are compressed in these areas. We can see a large peak of $p B_{\text {cme }}$ in case of GL while a much smaller one for TD. The smaller peak for TD flux rope could be related to its complex magnetic topology and subsequent quick loss of its own magnetic field by the process of magnetic dissipation and reconnection as it moves away from the Sun. Hence, $p B_{\text {cme }}$ decreases faster for TD over time. As the TD flux rope loses its own magnetic field, we get a negative $p B_{\text {cme }}$ at 48 minutes as can be seen in Figure 2(e). Similar result was described in Loesch et al. (2011). Hence, the plasma beta $(\beta)$ also becomes negative at the same locations for 


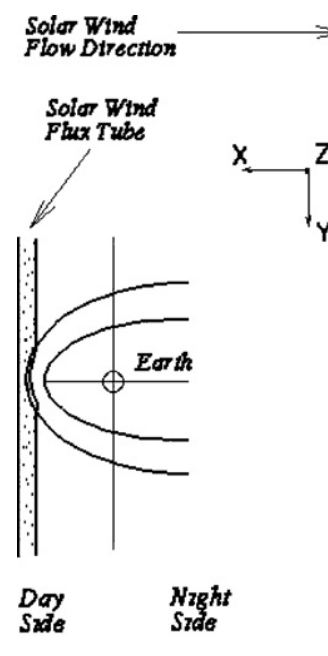

(a)

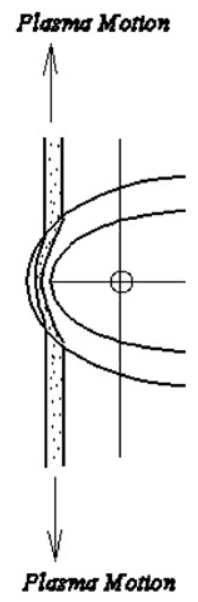

(b)

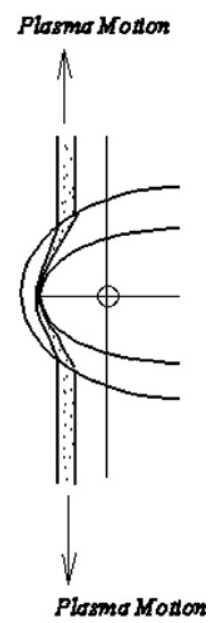

(c)

Figure 6. Plasma squeezing out process around the magnetosphere bow shock at successive times starting from (a) to (c). Adapted from Zwan \& Wolf (1976).

TD. In GL also, we observe a negative $p B_{\text {cme }}$ (negative again means lower than the steady state value) inside the PUC which we do not observe in case of TD. In TD, we actually observe the opposite where $p B_{\text {cme }}$ peaks inside PUC. Unlike TD, flux rope generally has low $\beta$ as $p B_{\text {cme }}$ inside the rope is high as can be seen for GL in Figure 2(b). Just ahead of the flux rope, we have a jump in $p B_{\text {cme }}$ which shows the existence of PDL, and then comes the PUC (with light yellow shade) having a jump in density and $\beta$. We find $\beta$ to be lower than 1 in all the regions except inside the PUC where it is much larger than 1. That means, the magnetic pressure dominates in all the places except inside the PUC where thermal pressure dominates because of its high density. $\beta$ is slightly higher inside the sheath than it is inside the flux rope where it is really small as can be seen in Figures 2(c) and (f). In the sheath, the plasma is compressed due to the shock. This creates a higher density of plasma and hence a slightly higher $\beta$ as well. Dasso et al. (2007) also reported $\beta$ to be lower than 1 inside the flux rope. Kilpua et al. (2009) reported in STEREO A observations from 2007 May 21 to 2007 May 24 that the flux rope has "typical" low density regions inside itself and is surrounded by the PUC region with $\beta$ greater than 1 . Interestingly, GL shows a very low $p B_{\text {cme }}$ while TD shows a very high $p B_{\text {cme }}$ right inside the PUC. Mostl et al. (2009) reported a CME sheath region having $\beta$ generally lower than 1 with a few peaks reaching above unity.

\section{DISCUSSION AND CONCLUSIONS}

Both PDL and PUC are important features of the CME sheath structure. PUC, especially, in the lower corona is very high in magnitude. This high PUC exists for a few reasons. One reason is that $V_{\mathrm{EX}}$ is large close to the Sun, as the CMEs are ejected with high internal pressure which decreases gradually as the CME moves further away from the Sun. Also, the solar wind density is higher in this region so that piled-up mass becomes higher as well. An additional contributing factor could be that we used a lower polytropic index $\gamma$ of 1.1 in our simulation. This drives a stronger shock in the region. We observe comparable propagation and deflection speeds in both TD and GL simulations. This makes $V_{\mathrm{EX}}$ predominantly responsible for the PUC formation at any stage. As we observe higher $V_{\mathrm{EX}}$ for GL than in TD in the lower corona, GL produces much larger PUC than TD. Also, the GL CME having larger size with larger initial magnetic pressure produces wider sheath and PUC area. Previous works, such as Erkaev et al. (1995) discussed analytical MHD theory of two extreme cases of (a) a purely expanding magnetic cloud and (b) a purely propagating magnetic cloud. They analytically derived a condition for the formation of a depletion layer, that we also found in our simulation, ahead of their magnetic cloud. In case of (b), they found the condition for a pile-up of plasma. As discussed in our paper, our simulation of CMEs are a combination of propagation and expansion of flux ropes, and we do observe the pile-up. A major difference is that we did not assume, as Erkaev et al. (1995) did, that the summation of the magnetic and thermal pressures is constant along the normal direction through the cloud.

Farrugia et al. (1997), using ISEE 3 and Interplanetary Monitoring Platform 1978 September 8 data and Farrugia et al. (2008), using ACE 2003 November data, observed that the defining characteristics of PDL are an increase of magnetic field strength and a simultaneous decrease of density adjacent to the front side boundary of MC. We also observe PDL in both of our simulations right ahead of the flux rope region where the magnetic fields are compressed and enhanced while the plasma are squeezed out from that region. Through the depletion of plasma, this region loses its thermal pressure and hence a lower $\beta$ region results. On the other hand, PUC having high density generally experiences high $\beta$. While Farrugia et al. (1997) showed a density jump from the PDL to PUC region of a factor of about 2.5 from ISEE 3 data, in our case, GL shows this factor to be about 2 and TD about 5 .

It is to be noted that although the exact values of the compression ratios for the shock and the PUC are dependent on the background solar wind, our results are general. Here we used a background solar wind heated with adiabatic index $\gamma<5 / 3$ so that the compression ratios for the forward shock and the PUC are greater than 4 . The compression ratios for the forward shocks have been observed to be between 1.1 and 2.8 in the white light coronagraphic images in the lower corona (Vourlidas \& Ontiveros 2009). However, our results still hold and indicate that whenever the expansion speeds are large, PUC will be large in the lower corona. We also expect that the PUC will contribute 
to the SEP (solar energetic particle) acceleration. We predict that, a massive and fast expanding $\mathrm{CME}$, producing a wider PUC, will also produce more SEPs. Also, CMEs ejected in the streamer belt region encounter hotter and denser solar wind plasma ahead of themselves. Streamer belt region, having slower solar wind, should create lower deflection speed in front of the CME. Therefore, a CME with large expansion and propagation speeds, could produce more PUC and hence more SEPs in the region. It is perhaps indicative that studies such as Kahler \& Vourlidas (2005), showing SEP-rich CMEs are the ones which look brighter in the white light coronagraphic images and are more likely to be the streamer blowouts or follow a co-located CME within 12-24 hours. The enhanced brightness suggests that large latitudinal and/or longitudinal extents are needed for fast CMEs to produce SEPs. This is consistent with PUC playing an important role in SEP-rich events.

So far as observed data close to the Sun is concerned, we are aware of the fact that this analysis is mostly based on two simulated CME-driven sheath and shock structures and comparing them with the actual data further away from the Sun, mostly near 1 AU. Hence, admittedly, we need more verifications with the actual coronagraphic data. Only since 2003, LASCO observations showed that white light coronagraphs can detect CME-driven shocks close to the Sun (review paper by Vourlidas \& Ontiveros 2009). Vourlidas \& Ontiveros (2009) also observed the so called CME "double front," one fainter and the other brighter front, in their running-difference coronagraphic images. While the fainter front indicates the shock front where the density enhancement is less, the brighter front, with higher density compression, indicates the coronal mass pile-up in front of the flux rope. Authors are not aware of any clear coronagraphic measurement of this brighter loop, which we define as PUC, in terms of density measurements so that we can compare our results with observations. Lynch et al. (2004) showed a rim structure in front of their flux rope in their breakout simulation. This rounded structure could be considered to be same as the PUC although their simulation did not include the background solar wind or any form of coronal heating.

The presence of a combination of PDL and PUC means two opposing phenomena are working in tandem here. On one hand, the flux rope pushes the plasma ahead of it and we see a PUC of high density. On the other hand, the CME also pushes the magnetic field lines ahead and compresses it so that we get a plasma depletion region with low density. These two opposing phenomena go hand in hand and might try to limit one another.

Manchester et al. (2005) reported a post-shock compression found in their simulation with GL-type FR. The FR was initially embedded in the helmet streamer under a bimodal solar wind atmosphere, and the interaction of the CME-driven shock with the bimodal solar wind forms a dimple in the streamer belt. As a result, the shock deflects the solar wind flow from the higher latitude toward the equator which produces a substantial post-shock compression. The mechanism of this post-shock compression appears to be different from our PUC as the flux rope/CME, in our case, displaces and pushes the plasma in the front to form this PUC. No deflection of flow due to the presence of a dimple appears to be creating this compression in our case. Liu et al. (2010) have also reported similar post-shock compression and PDL.

The high-density regions between the PUC and the forward shock could facilitate the accelerations of charged particles in the lower corona. Our next goal is to explore this problem estimating the acceleration of charged particles following magnetic field lines.

The authors thank the use of the Columbia and Pleiades clusters in NASA Ames. This research was supported by the NSF CAREER Grant ATM 0747654.

\section{REFERENCES}

Burlaga, L. F. 1988, J. Geophys. Res., 93, 7217, A7

Cohen, O., et al. 2007, ApJ, 654, L163

Dal Lago, A., Schwenn, R., \& Gonzalez, W. D. 2003, Adv. Space Res, 32, 2637

Dasso, S., Nakwacki, M. S., Démoulin, P., \& Mandrini, C. H. 2007, Sol. Phys., 244, 115

Démoulin, P., \& Dasso, S. 2009, A\&A, 498, 551

Erkaev, N. V., Farrugia, C. J., Biernat, H. K., Burlaga, L. F., \& Bachmaier, G. A. 1995, J. Geophys. Res., 100, 19919

Evans, R. M., Opher, M., \& Gombosi, T. I. 2011, ApJ, 728, 41

Evans, R. M., Opher, M., Jatenco-Pereira, V., \& Gombosi, T. I. 2009, ApJ, 703, 179

Farrugia, C. J., Erkaev, N. V., Biernat, H. K., Burlaga, L. F., Lepping, R. P., \& Osherovich, V. A. 1997, J. Geophys. Res., 102, 7089

Farrugia, C. J., Erkaev, N. V., Taubenschuss, U., Shaidurov, V. A., Smith, C. W., \& Biernat, H. K. 2008, J. Geophys. Res., 113, A00B01

Gibson, S., \& Low, B. C. 1998, ApJ, 493, 460

Gopalswamy, N., Dal Lago, A., Yashiro, S., \& Akiyama, S. 2009, Cent. Eur. Astrophys. Bull., 33, 115

Gosling, J. T., Riley, P., McComas, D. J., \& Pizzo, V. J. 1998, J. Geophys. Res., 103,1941

Gulisano, A. M., Démoulin, P., Dasso, S., Ruiz, M. E., \& Marsch, E. 2010, A\&A, 509, A39

Kahler, S. W., \& Vourlidas, A. 2005, J. Geophys. Res., 110, A12S01

Kaymaz, Z., \& Siscoe, G. 2006, Sol. Phys., 239, 437

Kilpua, E. K. J., et al. 2009, Sol. Phys., 254, 325

Liu, Y., Davies, J. A., Luhmann, J. G., Vourlidas, A., Bale, S. D., \& Lin, R. P. 2010, ApJ, 710, L82

Liu, Y., Manchester IV, W. B., Richardson, J. D., Luhmann, J. G., Lin, R. P., \& Bale, S. D. 2008, J. Geophys. Res., 113, 1029

Liu, Y., Richardson, J. D., Belcher, J. W., Kasper, J. C., \& Skoug, R. M. 2006, J Geophys. Res., 111, A09108

Liu, Y. C-M., Opher, M., Wang, Y., \& Gombosi, T. I. 2011, A\&A, 527, A46

Loesch, C., Opher, M., Alves, M. V., Evans, R. M., \& Manchester, W. B. 2011, J. Geophys. Res., in press

Lynch, B. J., Antiochos, S. K., MacNeice, P. J., Zurbuchen, T. H., \& Fisk, L. A. 2004, ApJ, 617, 589

Lynch, B. J., Li, Y., Thernisien, A. F. R., Robbrecht, E., Fisher, G. H., Luhmann, J. G., \& Vourlidas, A. 2010, J. Geophys. Res., 115, A07106

Manchester, W. B., IV, Gombosi, T. I., Roussev, I., Ridley, A., De Zeeuw, D. L., Sokolov, I. V., Powell, K. G., \& Toth, G. 2004, J. Geophys. Res., 109, A02107

Manchester, W. B., IV, et al. 2005, ApJ, 622, 1225

Mostl, C., Farrugia, C. J., Temmer, M., Miklenic, C., Veronig, A. M., Galvin, A. B., Leitner, M., \& Biernat, H. K. 2009, ApJ, 705, L180

Owens, M., \& Cargill, P. 2004, Ann. Geophys., 22, 4397

Owens, M. J., Cargill, P. J., Pagel, C., Siscoe, G. L., \& Crooker, N. U. 2005, J Geophys. Res., 110, A01105

Riley, P., Lionello, R., Mikic, Z., \& Linker, J. 2008, ApJ, 672, 1221

Rouillard, A. P., Lavraud, B., Sheeley, N. R., Davies, J. A., Burlaga, L. F., Savani, N. P., Jacquey, C., \& Forsyth, R. J. 2010, ApJ, 719, 1385

Roussev, I. I., Sokolov, I. V., Forbes, T. G., Gombosi, T. I., Lee, M. A., \& Sakai, J. I. 2004, ApJ, 605, L73

Siscoe, G., \& Odstrcil, D. 2008, J. Geophys. Res., 113, 1029

Thompson, B. J., Plunkett, S. P., Gurman, J. B., Newmark, J. S., St. Cyr, O. C., \& Michels, D. J. 1998, Geophys. Res. Lett., 25, 14, 2465

Titov, V. S., \& Démoulin, P. 1999, A\&A, 351, 707

Toth, G., et al. 2005, J. Geophys. Res., 110, A12226

Vourlidas, A., \& Ontiveros, V. 2009, in AIP Conf. Proc. 1183, Shock Waves in Space and Astrophysical Environments: 18th Annual Int. Astrophys. Conf., ed. X. A. \& G. Z. R. Burrows (Melville, NY: AIP), 139

Zwan, B. J., \& Wolf, R. A. 1976, J. Geophys. Res., 81, 1636 